\title{
Electric-field distribution in Au-semi-insulating GaAs contact investigated by positron-lifetime technique
}

\author{
C. C. Ling, Y. F. Shek, A. P. Huang, S. Fung, and C. D. Beling \\ Department of Physics, The University of Hong Kong, Pokfulam Road, Hong Kong, China
}

(Received 22 June 1998)

\begin{abstract}
Positron-lifetime spectroscopy has been used to investigate the electric-field distribution occurring at the $\mathrm{Au}-$-semi-insulating GaAs interface. Positrons implanted from a ${ }^{22} \mathrm{Na}$ source and drifted back to the interface are detected through their characteristic lifetime at interface traps. The relative intensity of this fraction of interface-trapped positrons reveals that the field strength in the depletion region saturates at applied biases above $50 \mathrm{~V}$, an observation that cannot be reconciled with a simple depletion approximation model. The data, are, however, shown to be fully consistent with recent direct electric-field measurements and the theoretical model proposed by McGregor et al. [J. Appl. Phys. 75, 7910 (1994)] of an enhanced EL2 ${ }^{+}$electron-capture cross section above a critical electric field that causes a dramatic reduction of the depletion region's net charge density. Two theoretically derived electric field profiles, together with an experimentally based profile, are used to estimate a positron mobility of $\sim 95 \pm 35 \mathrm{~cm}^{2} \mathrm{~V}^{-1} \mathrm{~s}^{-1}$ under the saturation field. This value is higher than previous experiments would suggest, and reasons for this effect are discussed. [S0163-1829(99)05008-0]
\end{abstract}

\section{INTRODUCTION}

Positron annihilation spectroscopy (PAS) has been widely used in semiconductor studies because of its sensitivity to open volume defects. ${ }^{1,2}$ When PAS studies are made of layered structures, such as semiconductor junctions, a variable energy positron beam is normally used to probe different depths and the annihilation quanta from the different layers relay information on defect structures in the layers. ${ }^{1}$ In order to perform a correct analysis, however, not only must the electric field present in the semiconductor be known, but some knowledge of the dynamics of the thermalized positron motion, expressed usually through the positron mobility, is also required. For the case of GaAs, diverse values of positron mobility, ranging from 32.5 to $880 \mathrm{~cm}^{-2} \mathrm{~V}^{-1} \mathrm{~s}^{-1}$, have been reported in the literature, and it has been suggested that the origin of this spread arises largely from uncertainties in band bending close to the semiconductor substrate surface. ${ }^{3}$ In addition to this, it is also to be expected that variations in positron shallow trapping at negatively charged acceptortype defects will be partially effective at room temperature, and cause some lowering of the mobility value. ${ }^{4}$

The depletion approximation, in which the electric field at a metal-semiconductor junction drops linearly with distance, is the common assumption that has often been used in the past to model the built-in electric field in most previous positron works on metal-semiconductor junctions. ${ }^{5-10}$ Positron diffusion in semi-insulating GaAs has previously been investigated by monitoring the fraction of positron drifted back to the metal-GaAs interface under the application of an electric bias. ${ }^{10}$ The data, analyzed with the assumption of the depletion approximation, gave a positron mobility of 70 $\pm 10 \mathrm{~cm}^{2} \mathrm{~V}^{-1} \mathrm{~s}^{-1}$ at $300 \mathrm{~K}$. However, some nonpositron works have recently revealed the failure of the depletion approximation in $\mathrm{Au}$-semi-insulating (SI) GaAs system, ${ }^{11-13}$ which report a lower than expected electric field and a more extended depletion region as compared to those predicted within the depletion approximation. McGregor et al. ${ }^{11}$ attributed this observation to an enhancement of the electroncapture cross section for the EL2 ${ }^{+}$center at electric fields above some critical value. Such an enhancement implies that a significant fraction of the EL2 ${ }^{+}$will be neutralized within the depletion region. In support of this view, a recent DBAR (Doppler broadening of annihilation radiation) experiment on $\mathrm{Au} / \mathrm{GaAs}$ and $\mathrm{Ni} / \mathrm{GaAs}$ structures employing monoenergetic positrons at different applied biases has revealed data supporting this model of incomplete ionization within the depletion region. ${ }^{3}$

In the present study, we investigate the electric field of the $\mathrm{Au}-\mathrm{SI}$ GaAs system with the use of PAL (positron annihilation lifetime) spectroscopy using a broad energy distribution of positrons from a radioactive source. Positrons are implanted into the sample from a ${ }^{22} \mathrm{Na}$ source, so that positrons were implanted up to a mean depth of about $50 \mu \mathrm{m}$, which is of similar dimension to the width of the field region under investigation. Different reverse biases are applied to the $\mathrm{Au}-\mathrm{SI} \mathrm{GaAs}$ structures so as to drift the implanted positrons back to the contact, and the intensity of the interfacial lifetime component is used to monitor the electric-field strength in the depletion region. A major objective has been to test various possible electric-field profiles, and the positron motion within them. In the first instance we test the standard depletion approximation model, and show it to give a poor description of our measurements. Then we turn to the model proposed by McGregor et al., ${ }^{11}$ and find it capable of giving a good prediction of the interfacial component's intensity over the whole bias range. Finally, we model the positron drift according to a parametrization of the recent experimental electric-field profile data of Castaldini et al. ${ }^{13}$ While the results are reasonable at high bias, experiment differs noticeably from theory in the midrange of applied biases, indicating the need for further investigations. 


\section{EXPERIMENT}

The semiconductor substrate used in this study was liquid-encapsulated Cholchralski-grown (SI) GaAs(100) single crystal obtained from Atramet Inc.. The thickness and resistivity of the substrate were $0.5 \mathrm{~mm}$ and 1.81 $\times 10^{8} \Omega \mathrm{cm}$, respectively. The substrate was cut into smaller pieces having a size of $1 \times 1 \mathrm{~cm}^{2}$, which were then subject to the same degreasing and etching procedures as used previously. ${ }^{3,8,14} \mathrm{~A} \mathrm{Au}$ disc with a diameter of $8 \mathrm{~mm}$ was electron beam evaporated onto both sides of the substrate at a pressure of $10^{-6}$ mbar. No subsequent annealing of the contacts was performed. The radioactive source used for the PAL spectrometry was a $30-\mu \mathrm{Ci}{ }^{22} \mathrm{NaCl}$ encapsulated by kapton foil. This source was sandwiched between the two sample pieces in a conventional manner, and electrical bias was applied to both sample pieces, and the two inner metal contacts that were exposed to the positrons were earthed. The outer two contacts were connected to a positive bias so as to set up an electric field in the sense required to drift positrons back to the internal pair of contacts. ${ }^{6,8,14}$ PAL spectra were collected for applied biases ranging from $-100 \mathrm{~V}$ up to $+240 \mathrm{~V}$, with a conventional fast-fast PAL spectrometer having a resolution of about $230 \mathrm{ps}^{8}$ A total of $4 \times 10^{6}$ coincident events was collected in each spectrum and then analyzed with POSITRONFIT software, ${ }^{15}$ correction being made for annihilations taking place in the kapton source foils.

\section{RESULTS AND ANALYSIS}

\section{A. Spectral analysis}

It was found that after background and source foil subtractions all of the spectra could be well represented by a three-exponential component fit, i.e.,

$$
n(t)=\sum_{i} \frac{N I_{i}}{\tau_{i}} \exp \left(-\frac{t}{\tau_{i}}\right)
$$

$N$ being the total number of spectrum counts, and $\tau_{i}$ and $I_{i}$ being the respective decay rate and intensity of the $i$ th component. A long $\sim 1000$-ps component of $\sim 0.04 \%$ intensity was found to exist in all the spectra. Following Ref. 16, this component was attributed to annihilations from the surface or the spaces in the sample-source sandwich assembly, and its lifetime and intensity were thus fixed for fitting all spectra so as to decrease any perturbation on the remaining free parameters.

The free fit results of the parameters $I_{2}, \tau_{2}$, and $\tau_{1}$ are shown in Fig. 1, plotted against the sample bias. It is seen that $I_{2}$ increases from $2 \%$ to $12 \%$ as the bias increases from 0 to $80 \mathrm{~V}$, after which it saturates. At negative and zero biases, $I_{2}$ remains nearly constant at $\sim 2 \%$. In the positivebias region, the increase of $I_{2}$ is accompanied by a decrease in $\tau_{1}$, whereas in other regions where $I_{2}$ is constant $\tau_{1}$ is also approximately constant. This behavior of $I_{2}$ and $\tau_{1}$ has been reported by others, ${ }^{17}$ and strongly suggests a defect trapping process at the metal-GaAs interface that increases as the applied bias is increased due to positron drift. $6,8,16,17$

In Fig. 1, although $\tau_{2}$ is observed to scatter about $400 \mathrm{ps,}$ there is clear evidence of an inverse correlation with $I_{2}$ caused by limited spectral information in the data fitting

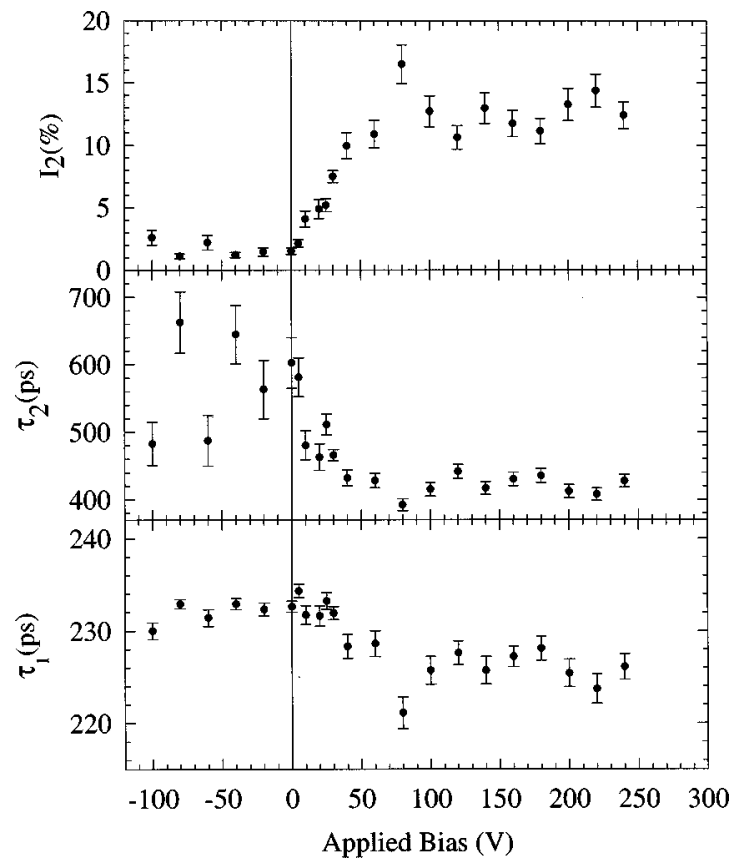

FIG. 1. Fitted values $I_{2}, \tau_{2}$, and $\tau_{1}$ as a function of the applied bias. All the parameters are treated as free parameters in the fitting process. A noticeable fitting correlation is seen between $I_{2}$ and $\tau_{2}$.

procedure. ${ }^{8}$ Since the one-defect trapping model ${ }^{18}$ would suggest a constant $\tau_{2}$, limited just by the free volume of the positron trapping sites, and the data can easily admit a constant $\tau_{2}$, the procedure adopted in this study was that of fixing $\tau_{2}$ so as to gain maximal information on the variation of $I_{2}$ by removing the undesirable correlation between $I_{2}$ and $\tau_{2} \cdot{ }^{8} \mathrm{~A} \tau_{2}$ value of $417 \mathrm{ps}$ was obtained by taking an average value of this parameter in the high-bias region. This value is close to that observed in other works (403 ps in Ref. 8, 410 ps in Ref. 16, and 464 ps in Ref. 17), and its magnitude indicates trapping into some open volume defect site of $\sim 5$ - $\AA$ radius, ${ }^{19}$ which may loosely be referred to as interface voids.

The fitted results of $I_{2}$, as obtained with $\tau_{2}$ fixed, plotted as a function of the applied bias, are shown in Fig. 2. $I_{2}$ is nearly constant at about $4 \%$ as the sample is in zero or negative bias, while it increases from $4 \%$ to about $13 \%$ as the bias is increased from 0 to $70 \mathrm{~V}$, and then saturated at $\sim 13 \%$ as the bias is further increased. The majority of the rise in $I_{2}$ has occurred by $\sim 50 \mathrm{~V}$. A similar saturation in $I_{2}$ has also been observed in Refs. 8 and 17, although with different saturation voltages (150 and $15 \mathrm{~V}$, respectively). It is noted that $I_{2}$ is not equal to zero under negative and zero biases a fact attributed to the natural built in field at the metalsemiconductor junction. ${ }^{8}$

\section{B. Positron drift under the depletion approximation}

In undoped SI GaAs, the EL2 deep donor that compensates for all residual shallow acceptors, thus rendering the material semi-insulating, is close to the midgap position, and its typical concentration is about $10^{16} \mathrm{~cm}^{-3}$. The shallow acceptors (such as the $\mathrm{C}$ impurity) that exist at lower concentrations $\left(\sim 10^{15} \mathrm{~cm}^{-3}\right)$ are thus essentially fully ionized, and the Fermi level is pinned close to the midgap $\left(E_{C}-E_{F}\right.$ 


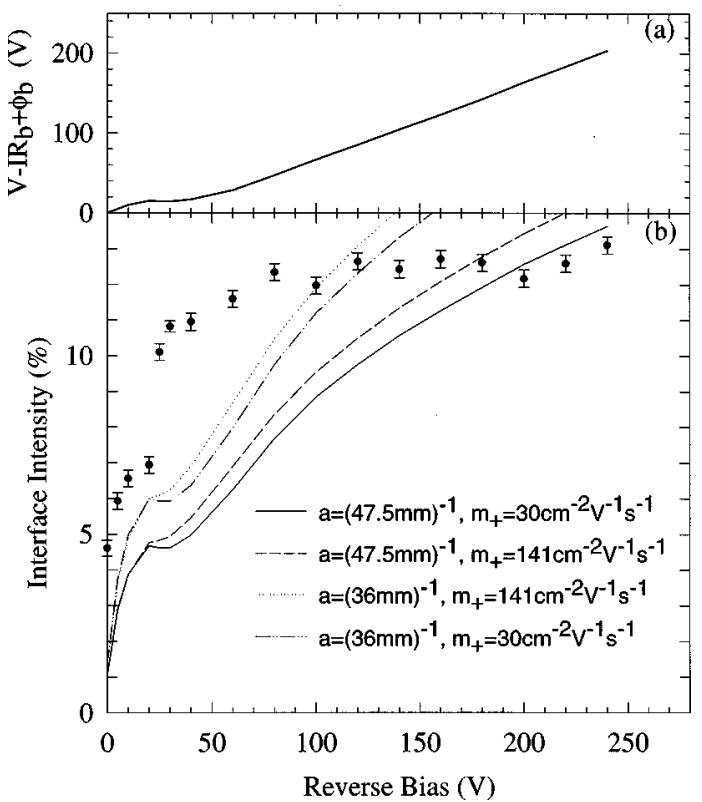

FIG. 2. (a) $\left[V-I R_{b}+\phi_{b}\right]$ as a function of the reverse bias, where $R_{b}=1.8 \times 10^{7} \Omega, \phi_{b}=0.8 \mathrm{eV}$, and $I$ is the measured current for each applied bias. (b) Fitted values $I_{2}$ as a function of the applied bias. The data points in the figure are obtained from the fitting of the spectrum by fixing $\tau_{2}=417 \mathrm{ps}$, so as to eliminate the correlation between $I_{2}$ and $\tau_{2}$. The modeled curves were obtained by the depletion approximation model and various values of $\alpha$ and $\mu_{+}$ indicated. The modeled curves cannot simulate the saturation of the $I_{2}$ data.

$=0.6 \mathrm{eV}$ for our sample). The electric field $\xi(x)$ and the electric potential $\varphi$ are determined at some depth $x$ into a planar sample by the Poisson equation ${ }^{20}$

$$
\frac{d^{2} \varphi}{d x^{2}}=\frac{d \xi}{d x}=\frac{e}{\varepsilon_{r} \varepsilon_{0}}\left[N_{\mathrm{DD}}^{+}(x)-N_{A}^{-}(x)-n(x)+p(x)\right]
$$

where $N_{\mathrm{DD}}^{+}$and $N_{A}^{-}$are the concentrations of ionized deep donor and shallow acceptor, respectively.

Under the normally employed depletion approximation, it is assumed that all the deep donors (i.e., EL2 in this case) in the depletion region are fully ionized, because they lie well above the bulk Fermi-level position. Thus an abrupt positively charged depletion region of width $W$, having a charge density equal to $\left(N_{\mathrm{DD}}-N_{A}\right)$, is formed and charge neutrality is maintained outside the depletion region. The solution of the electric field derives simply from Eq. (2) as ${ }^{20}$

$$
\xi(x)=\frac{e\left(N_{\mathrm{DD}}-N_{A}\right)}{\varepsilon_{r} \varepsilon_{0}}(W-x),
$$

the depletion width being given by

$$
W=\left(\frac{2 \varepsilon_{r} \varepsilon_{0}\left(\phi_{\mathrm{bi}}+V-I R_{b}\right)}{e\left(N_{\mathrm{DD}}-N_{A}\right)}\right)^{1 / 2},
$$

$\phi_{\mathrm{bi}}$ being the built-in contact potential, $V$ the applied external bias, $I$ the current passing through the sample, and $R_{b}$ the resistance of sample bulk.

In its simplest form the problem of determining the fraction of positrons that can drift against the competing annihi- lation process to an interface is given by the solution of the drift-diffusion annihilation equation

$$
\frac{\partial n}{\partial t}=\boldsymbol{\nabla} \cdot(n \mathbf{v})-\lambda n,
$$

in which the diffusion term has been omitted for simplicity, and because in the present case of determining $I_{2}$ we choose to work in the regime where drift exceeds diffusion. In Eq. (5), $n(x, t), v(x)$, and $\lambda$ are the positron density, drift velocity, and annihilation rate, respectively. This drift-annihilation equation must be solved subject to the time zero boundary condition, which for positrons emitted from the radioactive source is the close to exponential distribution ${ }^{21,22}$

$$
n(x, 0)=N \alpha \exp (-\alpha x),
$$

the constant $\alpha$ being known as the positron absorption coefficient.

The exact solution of Eq. (5) for a linearly varying electric field such as given in Eq. (2) is not one that lends itself to an easy analytical result. Thus in modeling it, it is normal to make the approximation of a constant electric field $\xi_{\text {I }}$ as given by the average value of $\xi(x)$ in this region:

$$
\xi_{\mathrm{I}}=\frac{1}{2} \frac{e N_{\mathrm{DD}}}{\varepsilon_{0} \varepsilon_{r}} W=\left(\frac{e N_{\mathrm{DD}}\left(\phi_{\mathrm{bi}}+V-I R_{b}\right)}{2 \varepsilon_{0} \varepsilon_{r}}\right)^{1 / 2},
$$

and in the constant electric field bulk region one simply has

$$
\xi_{\mathrm{II}}=I R_{b} /(d-W) .
$$

These electric fields are then considered to produce positron drift velocities $v_{\mathrm{I}}=v\left(\xi_{\mathrm{I}}\right)$ and $v_{\mathrm{II}}=v\left(\xi_{\mathrm{II}}\right)$ in the regions I and II, as given by the Schockley expression ${ }^{23}$

$$
v(\xi)=\sqrt{2} \mu_{+} \xi \frac{1}{\sqrt{1+\sqrt{1+(8 \pi / 3)\left(\mu_{+} \xi / v_{L}\right)^{2}}}}
$$

where $\mu_{+}$and $v_{L}$ are the positron mobility and the longitudinal sound velocity in GaAs. Spatial integration of Eq. (5) subject to the initial condition (6) now yields $n_{\mathrm{I}}$ and $n_{\mathrm{II}}$, the numbers of positrons in regions I and II, respectively, and to this may be added the number in the interface state $n_{2}:{ }^{8}$

$$
\begin{aligned}
\frac{d n_{\mathrm{II}}(t)}{d t}= & -\lambda_{b} n_{\mathrm{II}}-N \alpha v_{\mathrm{II}} \exp \left[-\alpha\left(W+v_{\mathrm{II}} t\right)\right] \exp \left(-\lambda_{b} t\right) \\
\frac{d n_{\mathrm{I}}(t)}{d t}= & -\lambda_{b} n_{\mathrm{I}}+N \alpha v_{\mathrm{II}} \exp \left[-\alpha\left(W+v_{\mathrm{II}} t\right)\right] \exp \left(-\lambda_{b} t\right) \\
& -N \exp \left(-\lambda_{b} t\right) f(t) \\
& \frac{d n_{2}(t)}{d t}=-\lambda_{2} n_{2}+N \exp \left(-\lambda_{b} t\right) f(t)
\end{aligned}
$$

where

$$
\begin{aligned}
f(t) & =\alpha \exp \left(-\alpha v_{\mathrm{I}} t\right) \quad\left(t<W / v_{\mathrm{I}}\right) \\
& =\alpha v_{\mathrm{II}} \exp \left\{-\alpha\left[v_{\mathrm{II}} t+\left(1-v_{\mathrm{II}} / v_{\mathrm{I}}\right) W\right]\right\} \quad\left(t \geqslant W / v_{\mathrm{I}}\right) .
\end{aligned}
$$


From these populations, after some manipulation, the intensity of the spectral component that decays as $\exp \left(-\lambda_{2} t\right)$ can be found as ${ }^{8}$

$$
\begin{aligned}
I_{2}= & \frac{\alpha v_{\mathrm{I}}}{\alpha v_{\mathrm{I}}+\lambda_{b}-\lambda_{2}}\left(1-\frac{\left(\lambda_{b}-\lambda_{2}\right)\left(1-v_{\mathrm{II}} / v_{\mathrm{I}}\right)}{\alpha v_{\mathrm{II}}+\lambda_{b}-\lambda_{2}}\right. \\
& \left.\times \exp \left[-\alpha W\left(1+\lambda_{b} / \alpha v_{\mathrm{I}}\right)\right]\right) .
\end{aligned}
$$

In order to compare the model with experiment, the following parameters and constants were taken: Based on the known bulk Fermi-level position $E_{C}-E_{F}=0.6 \mathrm{eV}$ and the Schottky barrier height of $\phi_{b} \sim 0.8 \mathrm{eV}$ for $\mathrm{Au}$ on GaAs, $\phi_{\mathrm{bi}}$ was taken as $0.3 \mathrm{eV} .^{8}$ The net charge density $N_{\mathrm{DD}}-N_{A}$ was taken as $[E L 2]-[C]=1.4 \times 10^{16} \mathrm{~cm}^{-3}$ given by the specifications of the manufacturer, $\varepsilon_{r}$ was taken as 13.2, and $v_{L}$ in Eq. (9) as $4.8 \times 10^{5} \mathrm{~cm} \mathrm{~s}^{-1} .^{8}$ The potential drop $V-\mathrm{IR}_{b}$ across the contact as a function as a function of the applied bias $V$ is shown in Fig. 2(a), and was calculated from measured current and taking $R_{b}=1.8 \times 10^{7} \Omega$. Regarding the positron absorption coefficient there is some disagreement on the correct value for GaAs. Using the formulation of Mourino, Löbl, and Paulin, ${ }^{21}$ who give $\alpha=2.8 \rho Z^{0.15} \bar{E}^{-1.19}$, where $\rho$ is the density, $Z$ is the atomic atom, and $\bar{E}$ is the mean $\beta^{+}$energy, one obtains $\alpha=(45 \mu \mathrm{m})^{-1}$. On the other hand, employing the equation of Brandt and Paulin ${ }^{22} \alpha$ $=16 \rho E_{m}^{-1.43}$, where $E_{m}$ is the maximum $\beta^{+}$energy, one obtains $\alpha=(50 \mu \mathrm{m})^{-1}$. To complicate matters further, Schrader $^{24}$ pointed out that the exponential form of Eq. (6) is strictly only valid for a collimated radioactive source and for an isotropically emitting source, a directional averaging should be carried out which reduces the effective value of $\alpha$ close to the contact. This view has to some extent found support from the work of Hansen, Linderoth, and Peterson, ${ }^{25}$ who, investigating the implantation profile experimentally, found that there was a superexponential fall at implantation distances less than $5.7 \mathrm{mg} \mathrm{cm}^{-2}(\sim 10 \mu \mathrm{m}$ in GaAs). Taking these effects into account, Shan et al. ${ }^{8}$ suggested an appropriate effective value of the implantation coefficient at the near-GaAs surface $\alpha_{\text {eff }}$ to be in the range $\sim(36 \mu \mathrm{m})^{-1}$ to (39 $\mu \mathrm{m})^{-1}$. It is thus in the present work that we take these two values as extremes in testing the model.

A comparison of the above model with experiment is shown in Fig. 2(b). In these plots two extreme values of 30 and $141 \mathrm{~cm}^{2} \mathrm{~V}^{-1} \mathrm{~s}^{-1}$ have been taken for the positron mobility so as to cover all reasonable values. It is observed that none of the modeled curves can give a good fitting to the experimental data. The reason is not difficult to find. Within the depletion approximation model, the depletion width increases from 0.25 to $4.6 \mu \mathrm{m}$, and the average electric field in the depletion region (i.e., $\xi_{\mathrm{I}}$ ) increases from 24 to 442 $\mathrm{kV} \mathrm{cm}^{-1}$, respectively, as the applied bias is increased from 0 to $240 \mathrm{~V}$. As positrons are implanted into GaAs to a mean depth 36-50 $\mu \mathrm{m}$, the increase in depletion width increases the fraction of positrons implanted into the high-field drift depletion region. Indeed it is noted from Eq. (11) that in the first approximation $I_{2}$ goes as $\alpha W$, i.e., in a $\sim V^{1 / 2}$ dependence. The increase of $I_{2}$ with the positron mobility (drift velocity) produced by the increase in the electric field is less marked, being a second-order effect. It thus follows that the depletion model cannot predict a saturated $I_{2}$ behavior for biases above $\sim 50 \mathrm{~V}$.

The same conclusions regarding the inappropriateness of the depletion approximation relate to the equivalent DBAR data taken on the identical $\mathrm{Au}-\mathrm{SI}$ GaAs system under applied bias. ${ }^{14}$ It was noted that the line-shape parameter $S$ decreased from about 0.5301 to 0.5281 as the applied bias increased from 0 to $50 \mathrm{~V}$, and then saturated at 0.5281 for higher applied biases. As with the present PAL study it was found that the depletion approximation model failed to describe the high bias saturation of the $S$ parameter.

In conclusion then, both the present PAL measurements and the DBAR measurements in Ref. 14 indicate that the intensity of the annihilation events coming from interfacial open volume sites increases first with increasing applied bias, but then this intensity saturates at biases above $50 \mathrm{~V}$. Since the dominant factor effecting the interfacial component intensity is the width of the high-field depletion region, the indication is that some limit on this width (or to a lesser extent the electric field within the width) is somehow occurring above biases $\sim 50 \mathrm{~V}$. Such a limitation is not consistent with the expectation of the model involving the depletion approximation as described above.

\section{Positron drift model with a saturating electric field}

As mentioned in Sec. III B, the most likely cause of the observed $I_{2}$ saturation at biases above $\sim 40 \mathrm{~V}$ is that the real depletion zone width $W$ is larger than predicted on the depletion approximation model. [By inference, the electric field would also have to be less since the integration of $\xi(x)$ over the depletion zone must always equate with the applied bias.] A few years ago McGregor et al. ${ }^{11}$ performed a set of a particle pulse height measurement experiments on Schottkycontacted GaAs radiation detectors and the results were also surprising in that they did not match with the depletion model based on a fully ionized EL2 donor. Indeed these workers found that the electric-field (depletion) zone extended at a much faster rate into the substrate (typically $\sim 1$ $\mu \mathrm{m}$ per $\mathrm{V}$ of applied bias). The discrepancy was explained in terms of an enhancement of the electron-capture cross section of the EL2 center as the electric field reached a critical value $\sim 10 \mathrm{kV} \mathrm{cm}^{-1}$. The dramatic increase in electroncapture cross section was seen as preventing significantly higher fields from existing in the sample since any ionized EL2 centers would tend to neutralize quickly at higher fields. ${ }^{11}$ Berwick et al., ${ }^{12}$ and more recently Castaldini et al., ${ }^{13}$ measured the electric-field profile of a semiinsulating GaAs radiation detector directly using the scanning surface potential technique. Their data clearly showed that the electric field at the metal-SI GaAs junction does not drop in the linear manner predicted by the depletion approximation, but indeed saturated at $\sim 10 \mathrm{kV} \mathrm{cm}^{-1}$, and remained approximately constant at this value until after some depth the field dropped to zero. Based on these observations $\mathrm{Hu}$ et $a .^{3}$ measured effective positron diffusion lengths in biased $\mathrm{Au}-\mathrm{SI} \mathrm{GaAs}$ and $\mathrm{Ni}-\mathrm{Si} \mathrm{GaAs}$ systems using depth scanning DBAR spectroscopy. Once again the data indicated a satu- 
rating electric field, the magnitude and width of which were consistent with the work of McGregor et al. ${ }^{11}$ and Berwick et al. ${ }^{12}$

Modeling the enhancement of the electron-capture cross section at high field in the manner proposed by McGregor et al. ${ }^{11}$ and as slightly modified by Hu et al. ${ }^{3}$ the ionized EL2 donor concentration is written in the form:

$$
N_{\mathrm{DD}}^{+}-N_{A}^{-}=\frac{N_{\mathrm{DD}}-N_{A}}{\left\{1+2 \exp \left[\frac{E_{F}-E_{\mathrm{DD}}+q \varphi}{k T}\right]\right\}\left\{1+\left(\frac{\xi}{\xi_{c}}\right)^{\gamma}\right\}}
$$

where $\xi_{C}$ is the critical field for the neutralization of the ionized EL2, and $\gamma$ (McGregor et al.'s $\alpha$ ) is a constant characterizing how fast the neutralization occurs with the increasing field. The first factor in the denominator of Eq. (12) just expresses the thermal ionization of the EL2 level based upon Fermi-Dirac statistics, while the second in a somewhat $a d$ hoc fashion expresses the sharp onset of $\mathrm{EL}^{+}{ }^{+}$neutralization for electric field $\xi$ exceeding the cross-section enhancement threshold at $\xi_{C}$. Determination of the electric-field profile with Eq. (12) is not simple because the form cannot be directly integrated since $N_{D D}^{+}-N_{A}^{-}$is a functional of both the electric field $\xi$ and the electrostatic potential $\varphi$. It is noted, however, that the first term in the denominator will only vary for low values of $\xi$ and $\varphi$ and that for a semi-insulator (i.e., where $n$ and $p$ are much less than the concentration of deep donors or acceptors) the relation between the electrostatic potential $\varphi$ and the electric field $\xi$ is known to $\mathrm{be}^{26}$

$$
\xi=\left[\frac{2 k T N_{\mathrm{DD}}}{\varepsilon_{r} \varepsilon_{0}}\right]^{1 / 2}\left\{\ln \left[\frac{\exp (-q \varphi / k T)+f_{\mathrm{DD}}}{1+f_{\mathrm{DD}}}\right]-\frac{N_{A}}{N_{\mathrm{DD}}} \frac{q \varphi}{k T}\right\}^{1 / 2}
$$

where $f_{\mathrm{DD}}=g_{\mathrm{DD}} \exp \left[\left(E_{F}-E_{\mathrm{DD}}\right) / k T\right], g_{\mathrm{DD}}$ being the degeneracy factor of the deep donor. Equation (13) of course assumes a field-independent electron-capture cross section, but such an assumption seems reasonable in the free-carrier tail region described by this equation. Armed with Eq. (13), the electrostatic potential $\varphi$ can be found for any $\xi$, thus effectively making $N_{\mathrm{DD}}^{+}-N_{A}^{-}$a function only of $\xi$. Numerical integration of Eq. (12) now gives the electric field profile $\xi(x)$ as follows:

$$
\frac{\varepsilon_{r} \varepsilon_{0}}{q} \int_{\xi(x)}^{\xi_{\max }} \frac{d \xi}{\left[N_{\mathrm{DD}}^{+}-N_{A}^{-}\right]}=x
$$

where $\xi_{\max }$, the maximum field at $x=0$, is given by

$$
\frac{\varepsilon_{r} \varepsilon_{0}}{q} \int_{0}^{\xi_{\max }} \frac{\xi d \xi}{\left[N_{\mathrm{DD}}^{+}-N_{A}^{-}\right]}=V+\phi_{\mathrm{bi}}-I R_{b}
$$

Electric-field profiles predicted by the above model at a variety of different biases are shown in Fig. 3(a), where we have put $\xi_{C}=10 \mathrm{kV} \mathrm{cm}^{-1}, \quad \gamma=10, \quad N_{\mathrm{DD}}=[\mathrm{EL2}]=1.5$ $\times 10^{16} \mathrm{~cm}^{-3}$, and $N_{A}=[C]=1 \times 10^{15} \mathrm{~cm}^{-3}$. The profiles are almost identical to those obtained by McGregor et al. ${ }^{11}$ The

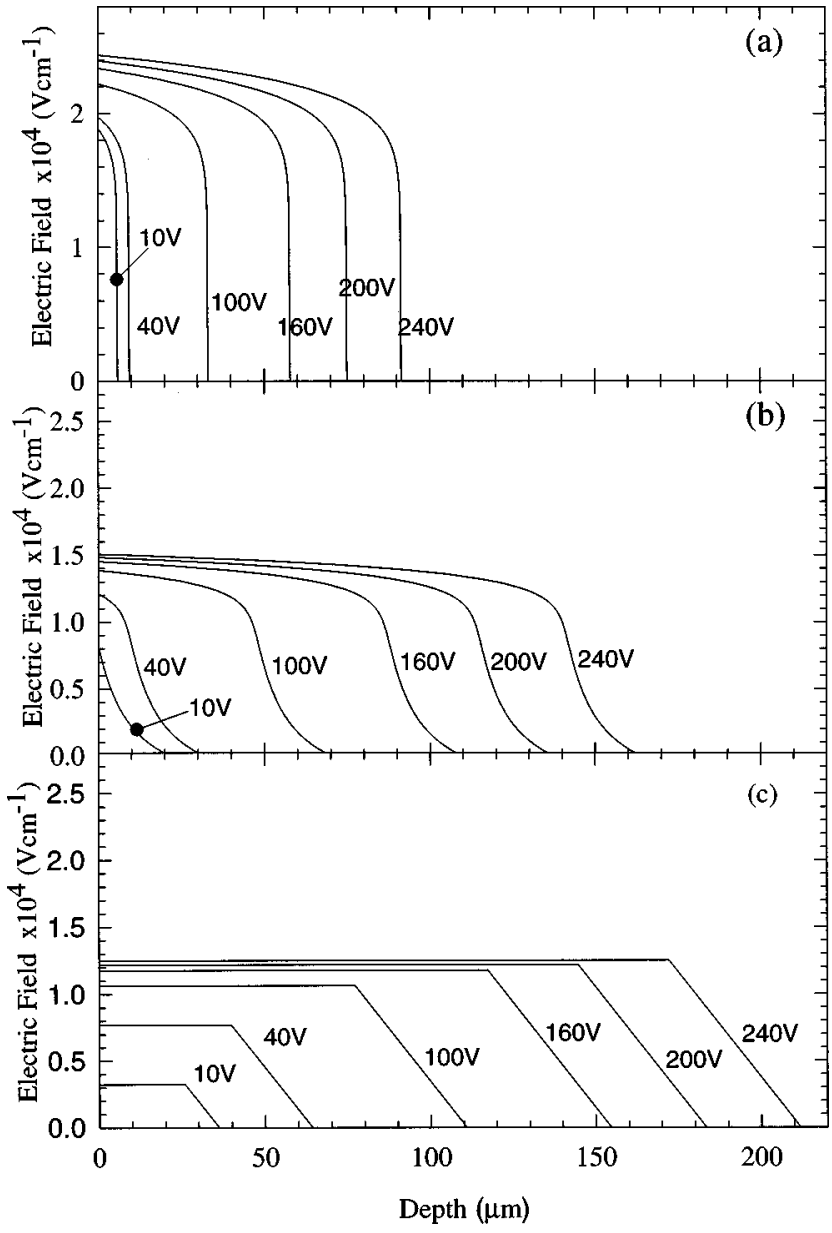

FIG. 3. Electric-field distribution $\xi(x)$ used in modeling the positron interface intensity $I_{2}$. (a) Model of Ref. 1 with $\left[N_{\mathrm{DD}}\right.$ $\left.-N_{A}\right]_{\mathrm{eff}}=1.4 \times 10^{16} \mathrm{~cm}^{-3}, \xi_{C}=10 \mathrm{kV} \mathrm{cm}^{-1}$, and $\gamma=10$. (b) Model of Ref. 1 with $\left[N_{\mathrm{DD}}-N_{A}\right]_{\mathrm{eff}}=1.4 \times 10^{13} \mathrm{~cm}^{-3}, \quad \xi_{C}=10 \mathrm{kV} \mathrm{cm}^{-1}$, and $\gamma=10$. (c) The linear parametrization of experimental data of Ref. 13

important point is that the decrease in electric field with depth is very different from the linear drop predicted within the depletion approximation. The electric field remains fairly constant up to a certain depth, and then drops abruptly. For example, a bias of $240 \mathrm{~V}$ now produces a depletion width of $\sim 90 \mu \mathrm{m}$ as compared to that of $5 \mu \mathrm{m}$ under the depletion approximation.

From Fig. 3(a), it is noted that the electric-field distribution can be divided into two regions, namely, a high-field region and a low-field bulk region, the latter occurring at depths beyond the abrupt boundary of the depletion region. Thus, in order to perform a fitting for the $I_{2}$ data, the same model used in the depletion approximation can be used, only with a slight modification. That is, the positrons in the highfield region I and low-field region II are drifted by the electric fields $\xi_{\mathrm{I}}$ and $\xi_{\mathrm{II}}$, respectively. The value of $W$ is taken as the position of the abrupt fall in electric field, while $\xi_{\mathrm{I}}$ is taken as the mean of $\xi(x)$ in the range $x=0$ to $W$. Positron velocities in the two regions are again described by Eq. (9), and the fitted $I_{2}$ curve obtained using Eq. (11), setting the implantation coefficient $\alpha=(47.5 \mu \mathrm{m})^{-1}$. The best-fitted curve is obtained as the positron mobility $\mu_{+}=100$ $\pm 10 \mathrm{~cm}^{2} \mathrm{~V}^{-1} \mathrm{~s}^{-1}$, which is within the range of the previ- 


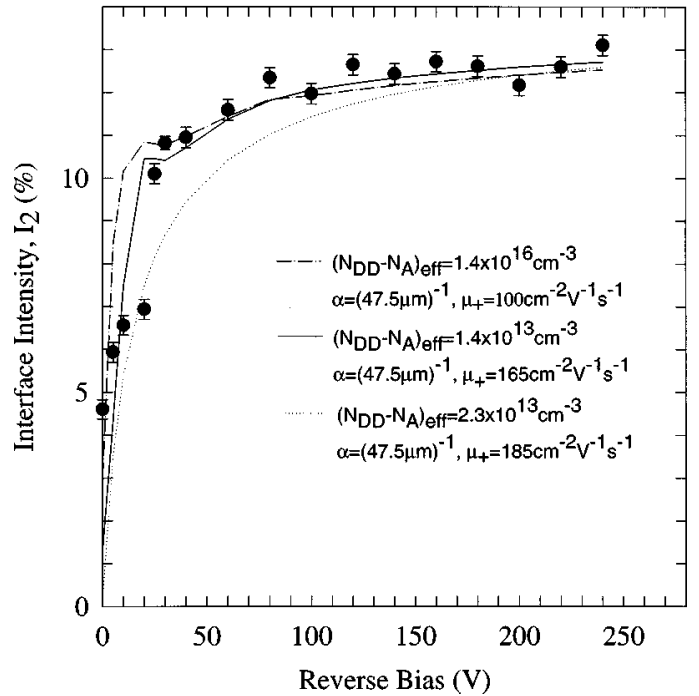

FIG. 4. $I_{2}$ data as a function of the applied bias shown with the saturated electric-field model fitted curves. The dot-dashed line is obtained from the electric-field profile shown in Fig. 3(a), i.e., $\quad\left[N_{\mathrm{DD}}-N_{A}\right]_{\mathrm{eff}}=1.4 \times 10^{16} \mathrm{~cm}^{-3}$ and $\mu_{+}=100 \mathrm{~cm}^{2} \mathrm{~V}^{-1} \mathrm{~s}^{-1}$. The solid line is obtained from the electric-field profile shown in Fig. 3(b), i.e., $\left[N_{\mathrm{DD}}-N_{A}\right]_{\mathrm{eff}}=1.4 \times 10^{13} \mathrm{~cm}^{-3}$, and $\mu_{+}$ $=160 \mathrm{~cm}^{2} \mathrm{~V}^{-1} \mathrm{~s}^{-1}$. The dotted line is obtained from the electricfield profile of Fig. $3(\mathrm{c})$ with $\left[N_{\mathrm{DD}}-N_{A}\right]_{\mathrm{eff}}=2.3 \times 10^{13} \mathrm{~cm}^{-3}$ and $\mu_{+}=185 \mathrm{~cm}^{2} \mathrm{~V}^{-1} \mathrm{~s}^{-1}$. In all fits the implantation parameter has been taken as $\alpha=(47.5 \mu \mathrm{m})^{-1}$. Increasing $\alpha$ to $(36 \mu \mathrm{m})^{-1}$ causes an equally good fitting, but with $\mu_{+}$scaled in inverse proportion.

ously reported values. ${ }^{3}$ The fitted curve is shown as the "dotdashed" line in Fig. 4 and is found to increase from about $3 \%$ at zero bias to about $13 \%$ as the bias is equal to $30 \mathrm{~V} . I_{2}$ is then found to saturate at about $12 \%$ at higher biases. The saturation of the measured $I_{2}$ value at $V>50 \mathrm{~V}$ can be well represented by the fitted curve although it gives a poorer fit to the data in the rising region (i.e., $V<50 \mathrm{~V}$ ).

Although the electric-field model obtained by putting $N_{\text {DD }}=1.5 \times 10^{16} \mathrm{~cm}^{-3}$ and $N_{A}=1 \times 10^{16} \mathrm{~cm}^{-3}$ can explain the saturation of $I_{2}$ data in the large applied bias region, the appearance of the electric-field distribution profile does not match well with experiment. Berwick et al. ${ }^{12}$ used the optical-absorption and scanning surface potential techniques to investigate the electric-field distribution in a semiinsulating GaAs radiation detector. Results obtained from both techniques were self-consistent, and also showed a region of nearly constant electric field for $x<100 \mu \mathrm{m}$ (see Fig. 2 in Ref. 12). Very similar results were obtained more recently by Castaldini et al. ${ }^{13}$ that confirm the measurements of Ref. 12. These results clearly show an approximately uniform electric field within the depletion region, but reveal one important difference, namely, that at the depletion width the fall to zero field is not abrupt. The data of Ref. 12 indicate a falloff distance of about $70 \mu \mathrm{m}$, whereas those of Ref. 13 suggest that the falloff distance is quite narrow $(\sim 14 \mu \mathrm{m})$ at low bias, extending in a linear fashion to about $30 \mu \mathrm{m}$ at higher biases. These falloff distances show that the net charge concentration, which we refer to as $\left(N_{\mathrm{DD}}\right.$ $\left.-N_{A}\right)_{\text {effective }}$, is $\sim 2 \times 10^{13} \mathrm{~cm}^{-3},{ }^{13}$ which is much lower than the value $\left(1.4 \times 10^{16} \mathrm{~cm}^{-3}\right)$ expected if all the EL2 centers were ionized. It is also of interest, that in the recent positron beam study on the metal-SI GaAs system, theory could only give a good fit to the data if a $\left(N_{\mathrm{DD}}-N_{A}\right)$ effective value of $\sim 1.4 \times 10^{14} \mathrm{~cm}^{-3}$ was taken. If the reasons for the quasineutral depletion region lies in some enhanced electron capture rate at high fields, we can speculate that the reason for the much lower degree of ionization observed at the edge of the depletion region finds its origin similarly. Johnson pointed out that the well-known transferred electron effect in GaAs would be expected to be operational at much lower fields (having a threshold field of $3.2 \mathrm{kV} \mathrm{cm}^{-1}$ for GaAs) and that this could possibly have the effect of increasing the electron-capture cross section if $L$-band capture was faster.

On the basis of the above observations, we tried another trial of fit to the present $I_{2}$ data by putting $\left(N_{\mathrm{DD}}\right.$ $\left.-N_{A}\right)_{\text {effective }}=1.4 \times 10^{13} \mathrm{~cm}^{-3}$. 3,13 The electric-field distribution generated from these parameters using the above model is shown in Fig. 3(b), and is in general agreement with the experimentally determined fields. ${ }^{13}$ The much slower falloff in the field than predicted by Ref. 11 [Fig. 3(a)] is apparent. The best fitted curve, taking the positron implantation coefficient $\alpha=(47.5 \mu \mathrm{m})^{-1}, \quad$ is obtained with $\mu_{+}$ $=165 \mathrm{~cm}^{2} \mathrm{~V}^{-1} \mathrm{~s}^{-1}$ and is shown as the solid line in Fig. 4. It is noted that he fitted curve gives a more reasonable agreement with the data at lower fields as well as still describing the high-bias saturation region well.

A final study we have carried out is to form a simple parametrization of the experimental electric field profile data of Castaldini et al. ${ }^{13}$ These authors wrote the depletion depth (defined by the $50 \%$ falloff in electric field) as

$$
W=W_{0}+k V
$$

where $W_{0}=24 \mu \mathrm{m}$ and $k=0.7 \mu \mathrm{m} \mathrm{V}^{-1}$. This allows us to write the plateau electric field that exists from $x=0$ to $x$ $=W-\delta / 2, \delta$ being the width of the electric field falloff region, as

$$
\xi=\frac{V}{W_{0}+k V} .
$$

The falloff in field from $x=W-\delta / 2$ to $W+\delta / 2$ is taken as linear as it also approximates, even at the lowest applied voltage, $5 \mathrm{~V}$, studied in Ref. 13. The electric-field profiles are shown for comparison in Fig. 3(c). The fit to the $I_{2}$ data as determined using these profiles is important because it should accurately mimic the real electric-field distribution in our samples, but, as seen from the fit of Fig. 4 (dotted line), the agreement is poor. In particularly the rise of $I_{2}$ in the bias range $0-100 \mathrm{~V}$ is much slower than it should be, although there is a tendency toward saturation which gives the fitted mobility as $185 \mathrm{~cm}^{-2} \mathrm{~V}^{-1} \mathrm{~s}^{-1}$. The reason for the disagreement is not well understood at the present time, but is probably related to the superior samples of Ref. 13, which had much lower reverse leakage currents. There are, of course, more model parameters in the electric-field distributions based on Eqs. (12)-(15), which in themselves would enable a better fitting over the parametrization based on experiment, which has essentially none. In spite of this uncertainty, there are indications that the modeling of the positron electric-field drift still demands further refinement. More specifically, Eq. 
(11) models drift over only two regions and ignores diffusion, and may therefore be too approximate.

We note that in all the above modeling, very high values $\left(100-185 \mathrm{~cm}^{2} \mathrm{~V}^{-1} \mathrm{~s}^{-1}\right)$ are obtained for the positron mobility in GaAs. Such values are generally much higher than found from diffusion length and Doppler shift measurements $\left(30-120 \mathrm{~cm}^{2} \mathrm{~V}^{-1} \mathrm{~s}^{-1}\right) .^{3}$ Attempts to modify the electric field or to cater for possible systematic errors in the $I_{2}$ data seem unable to account for the discrepancy. Indeed, the saturation of $I_{2}$ at $\sim 12 \%$ coupled with the fact that the positrons are drifting in a region of nearly uniform electric field, $\xi_{\text {sat }}$, necessarily implies a relatively high mobility. This follows because under these conditions Eq. (11) reduces to $I_{2}$ $=\alpha \mu_{+} \xi_{\text {sat }} /\left(\alpha \mu_{+} \xi_{\text {sat }}+\lambda_{b}-\lambda_{2}\right)$, and the mobility can be estimated accordingly since

$$
\mu_{+}=\frac{\lambda_{b}-\lambda_{2}}{\alpha \xi_{\mathrm{sat}}} \frac{I_{2}}{1-I_{2}} .
$$

With the electric field at the interface saturating in the range $(1.0-1.5) \times 10^{4} \mathrm{~V} \mathrm{~cm}^{-1}$ and an effective mean implantation range of $35-50 \mu \mathrm{m},{ }^{8}$ a mobility of $\sim 95 \pm 35 \mathrm{~cm}^{2} \mathrm{~V}^{-1} \mathrm{~s}^{-1}$ is deduced.

One possible explanation for a higher positron mobility is that positron shallow trapping occurs at room temperature, thus limiting the observed mobility, ${ }^{4}$ while under the saturated electric field the cross section for shallow trapping becomes diminished, thus raising the observed mobility. ${ }^{9}$ Another similar suggestion is that there is some reduction in the concentration of ionized defects or impurities in the depletion region, thus leading to less impurity scattering. An obvious example of this is in fact the EL2 defect, which takes on a reduced level of ionization in the quasineutral depletion region. Positron beam diffusion length experiments would not be so sensitive to this type of phenomenon, since they indirectly infer the mobility (which has to be assumed electric-field independent) from the diffusion coefficient. ${ }^{3}$ In the same context, it must also be recognized that our present understanding of the current transport at the metal-SI GaAs junction is far from complete. It is known, for example, that the reverse current through the junction is anomalously high if considered to be purely thermionic. ${ }^{27}$ Recently Santana and Jones suggested that since SI GaAs is a relaxation semiconductor, the generation current through some deep intermediate level is likely operative in enhancing the reverse current. $^{28}$ The change in charge state of some such generation-recombination center close to the interface could be important with regard to positron motion. These ideas also tie in with the recent work of Mazzer $e t$ al. who detected marked lateral variations in current transport across the metal-SI GaAs interface as if the density of interface states was also varying laterally. ${ }^{29}$ The neglect of these strong lateral variations in the electric-field modeling is another likely source of error in the present work that must be rectified in future studies.

\section{CONCLUSIONS}

PAL spectroscopy measurements have been performed on a $\mathrm{Au}$-semi-insulating $\mathrm{GaAs}$ system, in which positrons implanted from a ${ }^{22} \mathrm{Na}$ source have been drifted back to the positron implanted side by an application of electric bias. The longer lifetime component in the spectra, attributed to positrons that have drifted to the interface, has an intensity that increases with increasing reverse bias and which saturates at about $12 \%$ for biases above $50 \mathrm{~V}$. We have shown that attempts to describe this behavior using the standard metal-semiconductor depletion approximation fail, and cannot explain the observed saturation. In contrast, when we use the model of McGregor et al. ${ }^{11}$ that features an EL2 ${ }^{+}$neutralization dependent on the electric field, such that above the critical field $\xi_{C} \sim 10-15 \mathrm{kV} \mathrm{cm}^{-1}$ a quasineutral region develops behind the Schottky-like junction, then good agreement with the experimental data can be achieved. In particular the saturation observed in the intensity of the interfacial component above $\sim 50-\mathrm{V}$ reverse bias can be well explained. In agreement with the surface profile data of Berwick et al. ${ }^{12}$ and Castaldini et al., ${ }^{13}$ we find a better description of the data if we do not allow the electric field to drop abruptly to zero at the edge of the depletion region. Our data are consistent with a net charge density $\sim(1-2) \times 10^{13} \mathrm{~cm}^{-3}$ within the falloff region. Surprisingly, when we perform the positron drift modeling on a direct parameterization of the data of Castaldini et al., a less than perfect fit to the data is found in the midbias range of $30-150 \mathrm{~V}$, although the high-field saturation is as expected. The reasons for the discrepancy are not well understood. It is possible that some of the discrepancy is due to the oversimplified model describing the positron drift, but other explanations have also been forwarded such as the neglect of the lateral variation of the electric field.

While the present study has revealed the need for further investigations to explain fine structure in the positron drift experiment in the midbias range, it must not be overlooked that we now have a good understanding of the general shape of the interface intensity variation. It is now clearly understood that, as the bias increases beyond $40-50 \mathrm{~V}$, the electric field at the positron injecting contact is saturating. At the same time, since the depletion region expands at the rate of $\sim 0.7 \mu \mathrm{m} \mathrm{V}^{-1}$, the electric field covers the whole range of positron implantation $(35-50 \mu \mathrm{m})$. Application of further bias can neither affect the drift velocity of the positron nor the region of positron capture, and the interface intensity remains constant.

An interesting finding of the present work is that with the use of the saturated electric-field model, the positron mobility is estimated to be $\sim 95 \pm 35 \mathrm{~cm}^{2} \mathrm{~V}^{-1} \mathrm{~s}^{-1}$ which is higher than other recent estimates. We have suggested that perhaps a mobility in this range is a more accurate estimate of the correct mobility, and that other factors such as positron shallow trapping and impurity scattering are operative at room temperature and low fields which lower the value to $30-50$ $\mathrm{cm}^{2} \mathrm{~V}^{-1} \mathrm{~s}^{-1}$. At higher saturation fields either the reduction in the shallow trapping cross section or the number of ionized scattering centers could have the observed mobilityraising effect.

\section{ACKNOWLEDGMENT}

This work was financially supported by the Committee on Research and Conference Grants, The University of Hong Kong. 
${ }^{1}$ P. J. Schultz and K. G. Lynn, Rev. Mod. Phys. 60, 701 (1987).

${ }^{2}$ M. J. Puska and R. M. Nieminen, Rev. Mod. Phys. 66, 841 (1994)

${ }^{3}$ Y. F. Hu, C. C. Ling, C. D. Beling, and S. Fung, J. Appl. Phys. 82, 3891 (1997).

${ }^{4}$ K. Saarinen, P. Hautojärvi, A. Vehanen, R. Krause, and G. Dlubek, Phys. Rev. B 39, 5287 (1989).

${ }^{5}$ J. Mäkinen, C. Corbel, and P. Hautojärvi, Phys. Rev. B 43, 12114 (1991).

${ }^{6}$ R. I. Simpson, M. G. Stewart, C. D. Beling, and M. Charlton, J. Phys.: Condens. Matter 1, 7521 (1989).

${ }^{7}$ J. Mäkinen, C. Corbel, P. Hautojärvi, and A. Vehanen, Phys. Rev. B 42, 1750 (1990).

${ }^{8}$ Y. Y. Shan, H. L. Au, C. C. Ling, T. C. Lee, B. K. Panda, S. Fung, C. D. Beling, Y. Y. Wang, and H. M. Weng, Appl. Phys. A: Solids Surf. 59, 259 (1994).

${ }^{9}$ H. L. Au, C. C. Ling, B. K. Panda, T. C. Lee, C. D. Beling, and S. Fung, Phys. Rev. Lett. 73, 2732 (1994).

${ }^{10}$ Y. Y. Shan, P. Asoka-Kumar, K. G. Lynn, S. Fung, and C. D. Beling, Phys. Rev. B 54, 1982 (1996).

${ }^{11}$ D. S. McGregor, R. A. Rojeski, G. F. Knoll, F. L. Terry, Jr., J. East, and Y. Eisen, J. Appl. Phys. 75, 7910 (1994).

${ }^{12}$ K. Berwick, M. R. Brozel, C. M. Buttar, M. Cowperthwaite, P. Sellin, and Y. Hou, Mater. Sci. Eng., B 28, 485 (1994).

${ }^{13}$ A. Castaldini, A. Cavallini, L. Polenta, C. Canali, C. del Papa, and F. Nava, Phys. Rev. B 56, 9201 (1997).

${ }^{14}$ C. C. Ling, Y. Y. Shan, B. K. Panda, S. Fleischer, C. D. Beling, and S. Fung, Appl. Phys. A: Solids Surf. 60, 545 (1995).

${ }^{15}$ P. Kirkegaard, M. Eldrup, O. E. Mogenson, and N. J. Pederson,
Comput. Phys. Commun. 23, 307 (1981); P. Kirkegaard, N. J. Pederson, and M. Eldrup (unpublished).

${ }^{16}$ Y. Y. Shan, C. C. Ling, A. H. Deng, B. K. Panda, C. D. Beling, and S. Fung, Phys. Rev. B 55, 7624 (1997).

${ }^{17} \mathrm{~W}$. Yu and B. Cao, in Proceedings of the 9th International Conference on Positron Annihilation, edited by Z. Kajcsos and C. Szeles [Mater. Sci. Forum 105-111, 591 (1992)].

${ }^{18}$ R. N. West, Positron Studies of Condensed Matter (Taylor and Francis, London 1974), p. 71.

${ }^{19}$ P. Hautojärvi, J. Heinio, M. Manninen, and R. M. Nieminen, Philos. Mag. 35, 973 (1977).

${ }^{20}$ S. M. Sze, Physics of Semiconductor Devices, 2nd ed. (Wiley, New York, 1981).

${ }^{21}$ M. Mourino, H. Löbl, and R. Paulin, Phys. Lett. A 71, 106 (1979).

${ }^{22}$ W. Brandt and R. Paulin, Phys. Rev. B 15, 2511 (1977).

${ }^{23}$ W. Shockley, Bell Syst. Tech. J. 30, 990 (1951).

${ }^{24}$ D. M. Schrader, in Positron and Positronium Chemistry, Studies in Physics and Theoretical Chemistry, edited by D. M. Schrader, and Y. C. Jean (Elsevier, Amsterdam, 1988), Vol. 57, Chap. 2.

${ }^{25}$ H. E. Hansen, S. Linderoth, and K. Peterson, Appl. Phys. A: Solids Surf. 29, 99 (1982).

${ }^{26}$ C. G. B. Garret and W. E. Brattain, Phys. Rev. 99, 376 (1955).

${ }^{27}$ Y. L. Luo, T. P. Chen, S. Fung, and C. D. Beling, Solid State Commun. 9, 714 (1997).

${ }^{28}$ J. Santana and B. K. Jones, J. Appl. Phys. 83, 7699 (1998).

${ }^{29}$ M. Mazzer, A. Cola, L. Vasanelli, M. De Vittorio, C. Pennetta, and L. Reggiani, Inst. Phys. Conf. Ser. 157, 539 (1997). 